the fact be accounted for on physiological grounds, and what light will anatomy afford us in the investigation?

Foville, in his quarto work on the Anatomy of the Brain, has described and figured in his tables the optic nerves deriving a large amount of nervous matter from the sensory track, which he traces to the posterior columns (sensory) of the medulla oblongata. Supposing this anatomist to be correct, the second pair are compound nerves of special sense and common sensation.

Foville says, likewise, that the olfactory pair receive a portion of fibres from the sensory track.

If other investigators should confirm the anatomy of the brain, as described by this author, the phenomena of spasmodic conjunctivitis can be readily explained, but much of Dr. Marshall Hall's view will be set aside.

There 'are, however, circumstances which render it very doubtful that the retina contains any sensory fibres; I do not know any facts which indisputably prove that that organ possesses common sensation, or is sensible to any other stimulus than that of light. Nor can we derive much assistance from studying it in a diseased condition; for, although retinitis is an acutely painful disease, the surrounding structures of the globe of the eye are too rapidly involved in the inflammation to allow of our locating the seat of pain with any satisfactory degree of precision.

When the eye is exposed to intense light, pain follows, but even here the precise structure in which the uneasy feeling is mani fested is not positively certain. With regard to the olfactory nerves possessing common sensibility, the experiments of Sir Charles Bell and others are strongly against such an assumption. I may close this paper by observing, that it appears to me modern neurologists have generally described the brain and spinal cord as composed of sepurate organs operating independently of one another, or, at least, such is the general impression made upon my mind when studying their labours; whereas no line of demarcation can be drawn anatomically between one portion of nervous matter and another; motor and sensory tracks are not only in juxtaposition, but united by the closest unity of structure, and that the correct ideal notion of the nervous centre is, that the brain and spinal marrow form one great compound organ, of which the separate parts never act alone, or entirely independently of each other, and that by keeping this before us many physiological and pathological phenomena will lose much of their difficulty and darkness. For the present $I$ leave the inquiry-How light impinging on the retina excites contraction of the iris?

Old Market-street, Bristol, Mar̀ch, 1845.

THE POST-MORTEM APPEARANCES IN THE BODY OF SARAH HART, MURDERED AT SALT-HILL.

By Henrx Montagu Champnexs, Esq. Surgeon, Slough.

ON Wednesday evening, January 1st, 1845, about seven o'clock, I was hastily summoned to see Mrs. Hart, living two hundred and forty yards from my house. I immediately went, and on enter. ing, f and her extended on the floor with her head on a pillow. The countenance had a dusky appearance, the eye; were brilliant the pupils equally dilated, and quite insensible to the stimulus of light. I applied my hand to the region of the heart, but found no pulsation. The only symptom of life, just after I entered, was a slight movement of the lower jaw. I could detect no characteristic odour in the mouth. The neighbours cou'd give no account, further than thit she was well at two o'clock the same day. About an ounce of blood was drawn from the arm, which was quite fluid, at nine r.M. the same evening.

I performed the post-mortem, eighteen hours aft $r$ death, in the presence of Mr. Pickering and Mr. Nordblad. On stripping the body, we carefully noticed the absence of all external injury or violence whatever; the back of the shoulders down to the buttocks $w$ as of a livid hlue; the rectum had been emptied during the last moments of life, the motion still adhering to the anus, to the extent of about three inches.

The labia externa and thighs were stained with menstrual fluid; the labia interna were injected, and of a purplish colour The extremit:es were extremely rigid, and the lower jaw depressed with very great difficulty.

Upon $\mathrm{ma}^{\prime}$ ing an incision through the integuments, I immediately perceived the odour of prussic acid. Mr. Pickering also distinctly recognised it; Mr. Nordblad could not. The cavities of thorax and abdomen were then exposed. There were old adhesions between the pleura pulmonalis and costalis of both sides, particularly of the right, but no effusion; the lung itself was healthy throughout, but there was slight venous congestion. The pericardium was natural, and contained no fluid; the heart was natural size, contained dark fluid blood in the right side, but not the slightest appearance of coagulum; the left side quite empty, and appeared as if washed. The valves were carefully examined, and found healthy; there was no aneurism of aorta. The viscera of the abdomen were then examined ; the liver, spleen, and kidneys, had no appearance whatever of disease; they all contained fluid blood, the latter had a pinkish appearance. A double ligature was applied to both ends of the stomach, and it was then removed, and its contents (twelve ounces two drachms) placed in a clean bottle, and consisted of partially digested food, having a strongly acid beery odour, but not the least smell of prussic acid could be detected ; the internal coat of the stomach presented no appearance worthy of notice. The stomach itself, with part of iutestines, was placed in a bladder.

The uterus and ovaria were removed for inspection; they presented an increased degree of vascularity. The uterus was perfectly healthy, but rather large, with its cavity containing a dark sanguinous (no doubt catamenial) fluid, but no coagulum. The ovaria were healthy, the right had an opening into the peritoneum, communicating with the cavity of a Graafian vesicle, which contained coagulated blood, but there was not a trace of yellow matter, (as stated by Dr. Robert Lee, at page 246 of the present volume of THE LANCET.) The bladder was healthy, capacious, empty, and quite flaccid. The arterial system generally empty, and the venous for the most part gorged with fluid blood.

The head was now opened; the vessels of the membranes contained dark fluid blood; the substance of the brain was natural; there was no extravasation of blood, or effusion of serum, either between the membranes, or in the substance, or in the cavities of the brain. I also recognised the odour of prussic acid here, and called Mr. Pickering's attention to it several times, but he could not convince himself of it.

With the exception of there being congestion of the lungs, Mr. Pickering's notes agree with this account in every particular. March 20th, 1845.

\section{CLINICAL CONTRIBUTIONS FROM PRIVATE PRACTICE.}

CASE OF OVARIAN DROPSY CURED BY PRESSURE, MERCURIALS, AND DIURETICS.

By IsaAc B. Brown, Esq. Consulting surgeon to the Paddington Lying-in Charity.

THe following case of ovarian dropsy treated by pressure, mercurials, and diureties, will be found strongly illustrative of $\mathbf{m y}$ views, as shewn by some cases published in The Lancer, May 4th, 1844.

I hope the serious attention of my profescional brethren may be drawn to a perusal of this case. It is most strongly marked as one of three cysts, each cyst possessing a fluid of different character or colour.

August 13th, 1844.-Dr. Ely, of Rochester, called on me, with a young lady, aged twenty-seven years, the patient of his father, Mr. Ely, an old and extensive practitioner in Chatham.

Dr. Ely stated that she had been gradually increasing in size since the age of puberty, but that no particular notice of this increase was taken until a few years since, when, being on the point of marriage, she came to town to stay with the friends of her intended husband; they noticed her unusual size, and took her to Dr. Merriman, who declared it was ovarian dropsy, and advised, therefore, that marriage should not take place, but ordered no remedial treatment. She subsequently consulted. Dr. Lee, and others, but no one advised any measures with a view to cure; and she believing nothing could be done, reconciled her mind to the malady, and seclud d herself from society, although she was naturally of a lively and cheerful disposition. Shortly after the publication of my cases in ThE LANCET, a friend sent the paper to her, and that, by the advice of Mr. Ely, prompted her application to me.

On examining her in the recumbent posture, I observed her body the size of a seven months' pregnancy, with an evident increase on the left side, but the tumour extended across the median line into the right iliae fossa. On carefully examining this tumour, $I$ could clearly trace its circumscribed edges, and distinctly feel fluid within its walls, and felt assured it was a case of ovarian dropsy. I could discover no bardened tumour or enlargement in connexion with the cyst. Her health was materially affected by the disease, and her mind was very desponding; still, on the whole, I considered it a favourable case for $\mathrm{my}$ plan of treatment, and she readily consented to try it, and by the kindness of some friends, she made arrangements for staying in town under their roof.

On examining per vaginam, I found os uteri perfectly healthy 
and menstruation had always been regular. I found her measurement around the body, midway between the umbilicus and pubes, was exactly thirty-seven inches. I ordered her the following pills and mixture:-

B. Blue pill, $3^{i}$; powdered opium, gr. vj. Make into twentyfour pills, two night and morning.

$R$ Acetate of potass, $3 \mathrm{v}$; tincture of squills, $3^{i}$; spirit of juniper, $\zeta^{i}$; bicarbonate of potass, $3^{i}$; water, $\zeta^{x i i}$. $A$ mixture ; two table-spoonfuls three times a day

To rub the mercurial ointm nt into the thighs every night, and to apply flannel bandage round the abdomen, as tightly as she could bear it; to take nouriching diet, and exercise in the air.

Aug. 20th.- Gums slightly affected. Continue everything as before; adding to the mixture, infusion of gentian, $३ i v$.

This plan of treatment was carefully continued, and it was soon found that the size of the abdomen decreased considerably; in all, up to Sept. $17 \mathrm{th}$, four inches. I now considered it a tavourable time to tap the cyst, and draw off the fluid. Her health was much improved, and her spirits more cheerful and buoyant.

Sept. 17 th. - In the presence of my excellent friend, Mr. Samuel Lane, and Mr. Ely, I placed her on the edge of the bed, resting her feet on a chair, and introduced the trocar midway between the umbilicus and pubes; immediately a dark + coloured albuminous fluid, exactly resembling coffee not quite clarified, freely escaped, when it suddenly stopped; I thought, at the time, from the bandage being too suddenly tightened above the trocar, thus pressing the walls of the cyst over the mouth of the instrument and preventing any further escape of the fluid, six pints of which had been drawn off; but on careful examination, I could feel another cyst at least, and I fancied I could make out two, but as the patient was faint, I placed her in bed, and applied, very tightly, the flannel bandage. She expressed herself as feeling more comfortable, and much relieved by the loss of the fluid; she measured 33 inches below the umbilicus,

$$
31 \frac{1}{2}, \text { over " above }
$$

I continued the same mixture, pills, and ointment, after the operation, as well as tight bandaging.

On measuring her, Sept. $27 \mathrm{th}, \mathrm{I}$ found ste had decreased an inch since the $17 \mathrm{th}$, and was much improved in health, really looking quite another person in the face, and she expresses herself as conscious of decreasing, and is most cheerful, and hopeful of being cured.

Nov. 27 th. - I find her measurement now is, as compared to what it was Sept. 17 th, an inch and a half less. It is now $31 \frac{1}{2}$ inches below the umbilicus, 31 over, and $30 \frac{1}{2}$ above; but this decrease has not been perceptible during the last three weeks, and as there has been no increase, and her health is good and her gums still under the influence of mercury, I determined to tap the cyst which I left at the last operation. Accordingly, assisted by Mr. Lane and my brother Guorge, I at once introduced the trocar, and a fluid much clearer than the last freely escaped. This fluid was transparent, and of a yellow-straw colour, in quantity about five pints; when this ceased to flow, I could distinctly feel on the left side another cyst containing fluid, and on introducing a director through the canula, I could feel its round smooth surface. I introduced the trocar again, without removing the canula, and passing it upwards and to the left side, readily punctured the cyst, and a fluid, six pints, of a coffee colour, much resembling the first, freely escaped. As I could now feel no more fluid in the cavity of the abdomen, I withdrew the canula and applied adbesive strapping, gave the patient some wine and water; then some napkins, doubled up and applied one over the other, were placed over the cysts, and firmly bandaged down with flannel bandage, passed four times around the body over the hips; and fearing it might slip up, on account of the roundness of the hip-bones, I placed a silk handkerchief round the thigh, first tacking it to the lower edge of the bandage, and then tied it at the hip; this was done on both sides. She was now placed in bed, desired to rub in the mercurial ointment to the thighs, and to continue the pills and mixture as before. She measures now $27 \frac{1}{2}$ inches below the umbilicus, and $25 \frac{1}{2}$ above.

28th.-Passed a good night; has had no pain, and is feeling quite well. Applied a towel over the flannel bandages, and pinned it firmly down. Ordered her mutton chop, and wine and water.

29th.-Passed a good night; no pain: no dejection; continue rubbing and medicine; took off the bandages and reapplied them tighter than before, placing more napkins over the first, and over the flannel bandages a towel, very firmly pinned down. To take a mutton chop and sherry-water.

30th.-Finding the bandages too loose, I removed them, and placed a layer of napkins, and then a bandage, and over that some more napkins and another bandage, pinning or stitching the bandage each time it passed round the body ; applied silk handkerchiefs as before. To take two aperient pills, and continue medicine.

Dec. 2nd.-Bandages removed a little; took them off; measures twenty-seven inches below and twenty-five above; applied napkins again, first donbling some in a square form, and pressing them firmly down over the cysts; then some oblong ones over these, and a flannel bandage as tightly as possible, and two silk handkerchiefs as before around the thighs.

4th.-Bandages loose; took them off, and reapplied them as before as tightly as possible; she measures as before. To use the ointment night and morning, and to keep the recumbent posture.

10th.-Replaced napkins and bandages. To get up and walk a little.

15th.-Replaced bandages; omitted blue pill and mercurial ointment, and increased the quantity of acetate of potash and tincture of squills in the mixture; measures as before.

23rd.-Took off bandages, and applied them without any napkins; fastened silk handkerchiefs, as before, to the lower edge, and passed them round the thighs. Continue mixture. She went to church yesterday, and is much better in health.

28 th. - Took off the bandages, and ordered her to wear a flannel belt; measures twenty-seven inches below and twenty-six above. Continue mixture.

Jan. 1st, 1845. - Examined the body carefully, could feel a substance in the p lvis towards the left side, which appeared like collapsed cysts. I could feel no fluid. I allowed $h+r$ to leave town the following day. To continue the mixture, and to visit me in ten days.

14th.-Came up to town from Chatham with Dr. Ely; considered herself quite well; her friends all mach pleased at her altered appearance and continued good health. I carefully examined her, as also did Mr. Lane, but we could not detect any fluid in the hardened substance still remaining in the pelvis. She measures twenty-seven and twenty-six inches as before.

Feb. 15th. - I have lately heard from her, and she states that she is still perfectly well; and Dr. Ely considers her to have decreased since her last visit to me.

Having heard it frequently asserted by professional men, that in ovarian dropsy there is no absorption of the fluid, and that diuretics have no influence on the disease, I determined to have all the fluid she drank cartully measured, as also all that passed from the bladder. By examining the following tables it will be seen that there is a very great increase in the quantity voided, compared to that taken. How much of this may be attributed to the duretics I caunot say; my own impression is, that diuretics do act, and are consequently useful, in the treatment of ovarian dropsy, when they are combined with mercurials; and I feel equally certain that absorption does take place by means of the veins of the cyst. It will be noticed that this increase is perfectly independent of the excretions of the skin: this must tend to strengthen my opinion.

TABLE I.

Sept. 19 to $\mathrm{S}$ ept. $21 .-4$ pints taken, 7 pints roided 22 to 24. -4 28. -9 29 to Oct. $3 .-9$ 8 to 24 to

Nov. 1 to 8 to 8.- 9 15. -12 $22 .-9$ $29 .-9 \frac{1}{3}$ 7. -9 $13 .-10$ $20 .-9$

$\begin{array}{ll}" & 6 \\ " & 14 \\ " & 13 \\ " & 15 \\ " & 16 \\ " & 15 \\ " & 15 \\ " & 15\end{array}$

An increase of 50 pints. TABLE II.

Dec. 3 to Dec. $6 .-6 \frac{\mathrm{l}}{2}$ pints taken, 9 pints voided

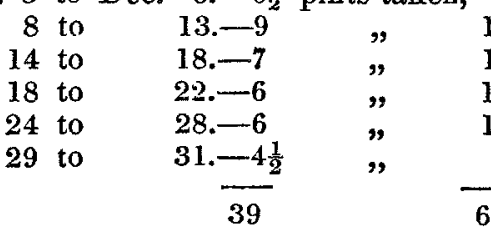

An increase of 24 pints.

This case must be looked upon as one which offered serious difficulties; and I candidly confess, that I hardly expected so happy a result, when I found, after the first tapping, that there were more cysts than one, as all my other cases were simple encysted; yet I think it must be acknowledged, the favourable termination of 
such a case is most conclusive of the efficacy of remedial agents, and will induce many to try this plan before having recourse to the abdominal section for the removal of the cyst.

I doubt not, many will maintain that there has not been sufficient time since the last tapping to warrant my considering this ease as cured; yet surely the history of it will shew that there are good grounds for so considering it; the gradual diminution before the first tapping, the decided decrease after it, and the absence of any increase since the last tapping, are all steady proofs of a decided influence over the fluid in the cysts; and furthermore, the health of the young lady, which was previously much impaired, is now restored; her menstruation continues regular, and she suffers no inconvenience from the collapsed cysts. I hope I shal hear no objection made to this case, as being one of doubtful dis ease; neither the age is too young nor the fluid too clear to leave any one an excuse for objecting to its character; and for the truth of what I have asserted, as to the nature of the disease, nature of fluid, and present state of patient, I can confidently refer to my friend Mr. Samuel Lane, who, from his uniform success in operating for the removal of the cyst by the median seetion, as well as from his sound surgical knowledge, must be considered a good au.hority. I may also mention, that Mr. Ely of Chatham, and Dr. Ely, of Rochester, will both cheerfully add their testimony to the truth of this case.

I cannot resist this opportunity of stating, that Miss $\mathrm{C}$ whose case I published in The LANcer, May 4th, 1844, continnes perfectly well, although nearly two years have elapsed since she was pronounced cured.

Oxford-square, Hyde-park.

OBSERVATIONS ON A CASE OF FATAL OVARIAN DISEASE. By ROBERT HARDY, Esq. Hull.

Is the month of August, 1843, I was called to officiate at the labour of Mrs. W-_, the lady of the Rev. N. W__, vicar of $\mathbf{S}$ - near this place. It was her first accouchement, the patient's age being twenty-seven or eight. She had been indisposed about twelve hours when the first examination was made. The outer parts and vagina were well lubricated, and disposed to relax; os uteri fully within the pelvic cavity, th $\mathrm{n}$, and $\mathrm{f}$ asily dilatable, and open to the size of a half-crown piece. Membranes thin, and protruding well during each pain. At the left posterior aspect of the pelvis, a considerable fulness was perceived, which was at the time supposed to consist of fæces in the rectum; presentation of the head in the right position, but resting on the os frontis; pelvic capacity ample. In two hours afterwards, the head was found in statu quo, though the pains in the interim had heen very efficient; the os uteri, also, was but little more dilated than when last examined.

It being evident some obstacle existed to the head's descent, a more careful examination of the swelling before alluded to was instituted, and it was found to consist of a firm tumour of definite form, (supposed to be ovarian,) and dipping into the pelvic cavity, as fir as the recto-vaginal pouch would admit of; it occupied nearly halt the circumference of the brim of the pelvis, and varied considerably in its degrees of density in different parts.

As all the maternal organs were so favourably disposed to delivery, I deemed it might assist us were we to rupture the membranes, which was accordingly done about half an hour after the examination of the tumour; and at the same period, a broad abdominal bandage $w$ as firmly applied. After the lapse of an hour, ma'ters were much as before. I had the patient now removed from bed, and placed between two chairs, as if sea ed on the night commode; in this position she remained about an hour and a nalf, during the whole of which, the pains were not only very frequent, hut also powerfully expellent. Still, at the end of this period, the head was advanced but very little; the scalp considerably corrugated; and, to my great mortification, the tumour not in the least displaced from its advanced position; but on the contrary, by the pressure from behind, had become more decidedly obstructive of the passage of the head into the pelvis.

During the last three hours, I had made several ineffectual attempts to push back the tumour; and I now became :pprehensive that we should ultimately have to reduce the child's head by perforation, as the space left for its descent was at least one half less than its natural dimensions. I stated my fears to the lady's husband, and urged on him the propriety of an early consultation on the case; this, however, he for the present declined, wishing me to act on my own judgment.

Before deciding on ulterior measures, I determined on making one further strenuous attempt to reduce the tumour ; for $t$., is pur pose, the patient was again removed to bed, her nates were con- siderably elevated, her shoulders depressed, and her face and abdomen inclined downward towards the bed. The right hand being well oiled, I passed it fully within the vagina, and waiting the sub idence of the next pain, I made firm pressure with the knuckles on the foetal head, pushing it pretty completely beyond the pelvic inlet; then, with the expanded fingers of the same hand, I exerted on the tumour a firm and steady pressure upwards, in the axis of he brim, which I was happy to find had some effect in altering its position. During the two succeeding pains, I was enabled to maintain the advantages already gained; advancing the tumour slowly upward in the intervals; after the third pain had gone off, to my great satisfaction I succeeded in elevating it quite to the pelvic brim, when it immediately slipped a way into the left hypochondrium. I still kept the hand within the vagina; the next pain advanced the head slightly, and after two or three others, it occupied the whole inlet. The hand was now withdrawn, the patient put in the usual position for delivery, and in l-ss than two hours, she was safely brought to bed of a very large and healthy female infunt. The placenta was cast off properly, and the recovery rapid and complete.

The first time this tumour appeared to inconvenience the patient again, was early in May of the present year, when I was called, in great haste, to visit her, as she was "labouring under obstruction of urine, and in great agony." On inquiry, I found Mrs. W- expected she was about four months advanced in gestation. The stoppage of urine was of fourteen or sixteen hours' duration; the hody was tumid and tender, and countenance expressive of great suffering; the attack of pain had been sudden, and the patient had passed her urine freely the preceding evening; she was also quite certain that she had used no violent exertion the previous day.

I stated to the patient my conviction that the cause of the present accident was the enlarced ovarian tumour, which had so seriously impeded the birth of the infant; my impression being, that it had produced the present symptoms by obstructing, to some extent, the brim of the pelvis, preventing the uterus from rising out of it into the abdominal cavity, depressing its fundus, and in this way inclining it backward, and ultimately, as the bladder filled, tilting this part of the uterus downward into the recto-vaginal pouch.

An examination per vaginam demonstrated a retroverted condition of the uterus. Three pints and a half of urine were drawn off, the patient placed on her knees, with the head downward, and, after some difficulty, the uterus was replaced in its proper position. The nates were ordered to be kepi considerably elevated, and the patient to preserve the horizontal posture some days; the urine to be passed as she laid, frequently. Next day I found all well; the urine had passed freely, and the bowels had been opened by castor oil. The recumbent position was persevered in, for the most part, during a fortnight; after which, the uterus was found to have risen fully into the abdomen, and no further present inconvenience was experienced from the presence of the tumour.

The second delivery nccurred about three A. M., on Saturday, the 19th of October; and so rapid was the process, that before my arrival, the infant had been expelled the uterus from fifteen to twenty minutes. The patient had had slight pains since ten o'clock of the evening preceding, but did not become seriously worse till about one A. M.; it is therefore clearly demonstrated that the ovarian tumour had been kept altogether out of the pelvic brim by the shoulder of the uterus, or the delivery could not have been thus rapid and facile. Much hæmorrhage had occurred (I suppose) after the birth of the infant, and yet continued; but this $I$ was enabled quickly to restrain by pressure on the uterus, which contracted weli. The placenta was ere long expelled, and we had no return of the flooding; nor did the patient seem to feel inconvenience afterwards, from the serious loss which she had sustained.

About forty hours after delivery, Mrs. W-_ began to complain of pain in the left hypochondrium, which steadily increased, till in a few hours it became most excraciating, the patient tossing violently about in bed, from her extreme agony. About three A M. on the Monday, I was sent for, and found her in great pain, which was described as of "a tearing colic kind," and identified by the patient as similar. in seat and character, to the pains experienced on a former occasion, from inflammatory obstruction in the bowels. For hor relief, there h.ıd already heen administered one ounce of castor oil also two doses of rhubarb and nagnesia, all of which the stomach had retained, but they had not as yet operated. Hot flannels had also been applied to the abdomen. 'The patient had had no shiverings, the lochı were plentiful, but there had been no attempt hitherto at lactation. The tongue was whitish and moist, skin cool, and pulse under 100 per minute. She expressed her conviction that complete relief would follow evacua- 